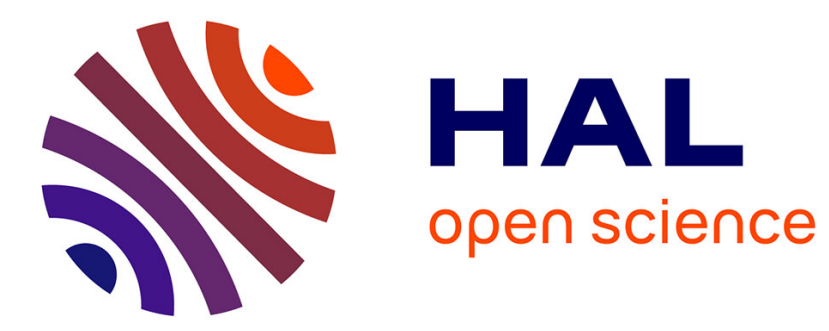

\title{
Mobilisations et animations autour des réductions d'intrants: stratégies d'intéressement des agriculteurs dans trois territoires franciliens
}

\author{
Aurélie Cardona, Claire Lamine, François Hochereau
}

\section{- To cite this version:}

Aurélie Cardona, Claire Lamine, François Hochereau. Mobilisations et animations autour des réductions d'intrants: stratégies d'intéressement des agriculteurs dans trois territoires franciliens. Revue d'Etudes en Agriculture et Environnement - Review of agricultural and environmental studies, 2012, 93 (1), pp.49-70. 10.4074/S1966960712001038 . hal-00939080

\section{HAL Id: hal-00939080 https://hal.science/hal-00939080}

Submitted on 30 Jan 2014

HAL is a multi-disciplinary open access archive for the deposit and dissemination of scientific research documents, whether they are published or not. The documents may come from teaching and research institutions in France or abroad, or from public or private research centers.
L'archive ouverte pluridisciplinaire HAL, est destinée au dépôt et à la diffusion de documents scientifiques de niveau recherche, publiés ou non, émanant des établissements d'enseignement et de recherche français ou étrangers, des laboratoires publics ou privés. 


\title{
Mobilisations et animations autour des réductions d'intrants : stratégies d'intéressement des agriculteurs dans trois territoires franciliens
}

\author{
Aurélie CARDONA*, Claire LAMINE **, François HOCHEREAU *** \\ * Auteur correspondant : EHESS GSPR / INRA Eco-innov, \\ 78850 Thiverval-Grignon, France \\ e-mail:aurelie.cardona@gmail.com \\ ** UR0767 Ecodéveloppement, INRA F-84914 Avignon cedex 9 \\ *** INRA SenS, F-77454 Marne-la-Vallée
}

Résumé - Dans un contexte où les remises en question de l'agriculture, notamment du système actuel de protection des cultures, vont croissantes, des acteurs variés, agricoles et non agricoles, se mobilisent autour de la réduction de l'utilisation des intrants de synthèse : on voit se développer une diversité de projets et de dispositifs pour amener les agriculteurs à faire évoluer leurs pratiques. A partir des cas de trois terrains franciliens, cet article analyse les registres d'argumentation et les dispositifs d'intéressement des animateurs territoriaux agricoles et non agricoles ayant pour objectif d'inciter à l'adoption de pratiques à plus faible niveau d'intrants en montrant la diversité et l'évolution des formes de conseil à l'œuvre. Il vise aussi à comprendre les effets de ce travail d'intéressement : conduit-il à un verdissement des pratiques ou plutôt à une écologisation globale ?

Mots-clés : agriculteur, réduction d'intrants, animateur territorial, argument, stratégie d'intéressement

\section{Mobilizations and local facilitations towards the reducing use of pesticides: Incentives strategies targeting farmers in the Paris area}

Summary - In a context where the current system of crop protection is strongly criticized, various actors (both from the agricultural and the non agricultural world) are getting mobilized towards pesticide reduction. A diversity of incentive strategies are emerging to encourage changes in farming practices. We analyze three case studies in the greater Paris area to understand the forms of argumentation and the incentive strategies of local facilitators to encourage transitions towards sustainable practices. Thus, we show the diversity and the evolution of the forms of advisor services. Then we analyze the effects of these incentive strategies. Do they lead to a greening or to an ecologization of farming practices?

Keywords: farmer, pesticide reduction, local facilitator, argument, incentive strategy

Classification JEL : Z00, Q15, Q16

Cette recherche est soutenue par l'attribution d'une allocation doctorale de la Région Ile-de-France et par le projet ANR POPSY (Systèmes de production de grande culture, environnement, politiques publiques). 


\section{Introduction}

Les critiques grandissantes quant à l'impact des intrants chimiques en agriculture et de récents dispositifs publics, tels que le Grenelle de l'environnement ou encore Ecophyto 2018, conduisent divers types d'acteurs, agricoles et non agricoles, à mettre en ouvre des actions visant à favoriser les pratiques économes en intrants. Le conseil agricole se transforme et de nombreuses collectivités territoriales financent des aides aux agriculteurs pour faciliter les conversions et le maintien de l'agriculture biologique (Ruault, 2006), encouragent l'approvisionnement local et biologique de la restauration collective $^{1}$, participent à la protection des ressources en eau à travers des actions d'incitation à la réduction des intrants chimiques et soutiennent des projets associatifs en faveur du développement durable (Barthe, 2009). Des associations ou collectifs issus de la «société civile non agricole ${ }^{2}$ se mobilisent, eux aussi, dans un contexte favorable aux questionnements sur l'impact environnemental des productions agricoles (nitrates, nuisances et pollutions engendrées par les élevages hors-sol) et sur les risques liés à l'emploi de différentes technologies (farines animales, OGM, pesticides). Ainsi, les interactions entre monde agricole et monde non agricole (agences de l'eau, élus locaux, collectivités territoriales, associations environnementales, riverains, etc.) se multiplient autour des questions de l'impact environnemental des pratiques agricoles (Lémery, 2003).

Il a déjà été montré que des interactions entre producteurs et consommateurs au sein de dispositifs de type AMAP ${ }^{3}$ peuvent favoriser l'adoption de pratiques plus durables même si cela ne concerne qu'un nombre limité d'agriculteurs (Lamine, 2005). D'autres travaux ont mis en évidence l'importance d'autres acteurs non agricoles pour la valorisation de pratiques agricoles plus écologiques (Candau et Ruault, 2005). Par ailleurs, au sein même du monde agricole institutionnel (chambres d'agriculture, syndicats professionnels...), il apparait que l'introduction d'exigences environnementales modifie largement le métier de conseiller (Brives, 2006). Plus largement, dans un contexte de redéfinition des contours et des pratiques du secteur agricole, le conseil agricole se déplace de la diffusion d'innovations techniques vers une fonction de

1 La loi du 27 juillet 2010 de modernisation de l'agriculture et de la pêche fait ainsi mention de la nécessité de développer les circuits courts et les relations de proximité entre producteurs et consommateurs, en particulier pour la restauration collective. Cf. Journal officiel du 28 juillet 2010. 2 La société civile est classiquement définie comme constituée par «l'ensemble des rapports interindividuels, des structures familiales, sociales, économiques, culturelles, religieuses, qui se déploient dans une société donnée, en dehors du cadre et de l'intervention de l'Etat. » (Quermonne, 1986). L'usage du terme « société civile non agricole » vise ici à désigner des acteurs caractérisés par leur volonté d'indépendance vis-à-vis de l'Etat et du monde agricole institutionnel (Chambres d'agriculture, syndicats agricoles, ....). La qualification «non agricole » a donc pour objectif de souligner l'engagement d'un nouveau type d'acteurs dans les questions de changement agricole.

3 AMAP : Association pour le maintien de l'agriculture paysanne ayant pour objectif de préserver l'existence et la continuité des fermes de proximité dans une logique d'agriculture durable [...], qui réunit un groupe de consommateurs et un agriculteur de proximité autour d'un contrat dans lequel chaque consommateur achète en début de saison une part de la production qui lui est livrée périodiquement à un coût constant. Le producteur s'engage à fournir des produits de qualité dans le respect de la charte de l'agriculture paysanne (extrait de la charte des AMAP, mai 2003). 
médiation dans des réseaux d'acteurs aux intérêts parfois antagonistes et une fonction d'accompagnement vers un avenir incertain (Lémery, 2006).

L'objectif de cet article est d'étudier à l'intervention de nouveaux acteurs dans le champ de l'animation agricole et de saisir en quoi ils participent à l'évolution des formes de conseil agricole dans un contexte d'écologisation, c'est-à-dire dans un contexte où le monde agricole est appelé, de manière croissante, à intégrer des contraintes environnementales.

Pour cela, nous nous intéresserons plus particulièrement à l'influence de la distance au monde agricole institutionnel sur les modalités de l'activité de conseil et sur les changements de pratiques des agriculteurs. Nous montrerons également l'importance de l'ancrage sur le terrain et dans le monde agricole local. Nous confronterons ainsi le rôle que peuvent jouer des dynamiques territoriales émanant d'organismes institutionnels agricoles - tel qu'une chambre d'agriculture - et d'associations issues de la société civile non agricole dans les trajectoires de changement des agriculteurs. Nous étudierons les différentes stratégies d'intéressement (Akrich et al., 1988) des agriculteurs mises en œuvre par ces organisations en nous concentrant sur les activités des animateurs de changement ${ }^{4}$ directement en contact avec les agriculteurs - activités basées sur le recours à certains types d'argumentation et l'emploi de dispositifs incitatifs. Cependant, il ne s'agira pas tant d'analyser la diffusion d'innovations ou de nouvelles pratiques agricoles dans un milieu a priori plus ou moins favorable, mais plutôt de saisir comment est suscité l'intérêt des agriculteurs pour des pratiques moins consommatrices en intrants en montrant l'importance de l'ancrage sur le terrain et dans le monde agricole local. Ainsi, nous tenterons de contribuer à la compréhension de la construction des mobilisations et des actions collectives autour de la question des réductions d'intrants en essayant de comprendre les processus par lesquels des acteurs divers se rassemblent autour d'un problème commun.

Pour saisir la diversité des stratégies d'intéressement de ces animateurs de changement et leurs effets sur les pratiques agricoles, trois terrains ont été considérés. Tous se situent en région Ile-de-France, et tous les agriculteurs rencontrés produisent majoritairement des céréales, même si certains ont diversifié leur activité. Un premier terrain d'étude se situe en Essonne, sur le plateau de Saclay, où les agriculteurs subissent une forte pression foncière et environnementale en raison de leur enclavement dans une zone périurbaine. Ici, un groupe de riverains ayant formé une AMAP se mobilise pour préserver les terres agricoles et promouvoir une agriculture plus respectueuse de l'environnement. Les deux autres terrains d'étude se situent en Seineet-Marne, dans des territoires qui constituent d'importantes réserves en eau approvisionnant l'agglomération parisienne. Dans ce département, en 2006, les acteurs concernés par la gestion de l'eau (acteurs agricoles locaux, organismes gestionnaires de l'eau, collectivités territoriales et services déconcentrés de l'Etat...) ont défini un plan départemental de l'eau (PDE). Certains de ces acteurs assument sa mise en œuvre sur

4 Sur les deux terrains seine-et-marnais, ces acteurs se nomment eux-mêmes « animateurs » tandis que sur le plateau de Saclay les acteurs se voient davantage comme des «facilitateurs ». Cependant, dans un souci de clarté et l'usage de la notion de «facilitateur » n'ayant été notée qu'une fois, on utilisera pour tous les acteurs le mot « animateur». 
des territoires prioritaires de protection et de reconquête de la qualité de l'eau. Les deux cas étudiés sont le bassin versant de l'Yerres, où l'animation en faveur des réductions d'intrants est assurée par la chambre d'agriculture, et celui de l'Ancœur où intervient une association locale d'usagers de l'eau. On peut ainsi distinguer les animations selon un gradient exprimant leur distance au monde agricole institutionnel et leur degré de compétence technique qui va de l'activité de membres d'une AMAP (Saclay), caractérisée par une faible professionnalisation ${ }^{5}$, à l'animation d'un conseiller technique dans une chambre d'agriculture (Yerres) fortement professionnalisée, en passant par l'action d'une association qui s'est professionnalisée au cours du temps (Ancœur).

Au point de vue méthodologique, nous nous appuyons sur 35 entretiens semidirectifs auprès d'agriculteurs et animateurs et dix réunions ou moments collectifs auxquels nous avons assisté, intégralement retranscrits afin d'analyser les ressorts argumentatifs des acteurs rencontrés et les confrontations de positions entre acteurs agricoles et non agricoles.

Après la présentation des trois territoires et des configurations d'acteurs les caractérisant, nous analyserons les différentes stratégies d'intéressement mises en œuvre. Pour cela, nous étudierons les registres d'argumentation et dispositifs incitatifs utilisés par les animateurs. Enfin, nous tenterons de saisir les effets de ces diverses actions sur les pratiques agricoles pour comprendre dans quelle mesure ces actions d'animations et ces politiques publiques entraînent une réorganisation des systèmes agricoles et si elles correspondent plutôt à une écologisation ou à un simple «verdissement » des pratiques agricoles (Deverre et de Sainte Marie, 2008).

\section{Trois territoires contrastés}

Sous l'effet de pressions environnementales croissantes, des formes de mobilisation très diversifiées apparaissent sur les trois territoires étudiés. Les modalités observées de mise en œuvre d'une animation territoriale peuvent être différenciées en fonction de l'origine de cette animation, à savoir plutôt la société civile ou plutôt les structures institutionnelles. Pour mieux comprendre ces différents types d'animations et leurs ressorts d'action, nous allons expliciter ici leur genèse, leur composition et leurs modes d'intervention.

\subsection{Une association locale dont la sphère d'action s'élargit progressivement}

Nous avons étudié le cas du plateau de Saclay à partir d'entretiens auprès d'acteurs du monde associatif (7), d'agriculteurs (8) et d'un suivi de divers moments collectifs sur une durée de 18 mois.

Depuis les années 1960, ce territoire enclavé est soumis à une forte pression foncière, et de nombreuses associations environnementales locales ont été créées suite à des projets d'urbanisation ou d'infrastructures routières sur des espaces naturels ou

5 La professionnalisation désigne ici la rémunération des activités d'animation. 
agricoles. Au début des années 2000, un groupe de riverains, interpellé par la mise en place d'un plan local d'urbanisme (PLU) menaçant les terres agricoles, a décidé de réagir également. Ce groupe, composé essentiellement de chercheurs, enseignants ou ingénieurs dans les établissements d'enseignement supérieur et centres de recherche environnant le plateau ou encore de cadres dirigeants dans les entreprises locales ou parisiennes, est peu en lien avec le monde agricole, mais il soutient la candidature de Pierre Rabhi 6 aux élections de 2002. Par le biais des rencontres avec le candidat, le groupe est largement sensibilisé aux notions d'échange et d'interdépendance entre citadins et agriculteurs. Dans cette perspective, les riverains décident de contacter les agriculteurs du territoire afin de mettre en place un partenariat permettant de faire face ensemble à la menace d'urbanisation. Suite à la réponse d'un seul agriculteur (sur les huit encore en activité), une AMAP est créé fin 2003, produisant, sur trois hectares ${ }^{7}$ d'une exploitation qui en compte 237, pommes de terre, lentilles et farine sans intrants de synthèse. Au-delà de l'objectif de production d'aliments, il s'agit de créer des liens forts entre agriculteurs et urbains et de faire évoluer les pratiques agricoles vers des pratiques plus respectueuses de l'environnement.

Dans la continuité des projets d'urbanisation des années 1960, l'Etat lance en 2005 une opération d'intérêt national (OIN) en vue d'aménager ce territoire, ce qui ravive la contestation locale. Lors de l'annonce de ce nouveau plan d'aménagement, un certain nombre d'acteurs à l'origine de l'AMAP sont devenus, aux côtés des anciennes associations environnementales, des acteurs incontournables se plaçant comme défenseurs légitimes des questions agricoles et du territoire. Ces mobilisations ont notamment contribué à inscrire la préservation de 2300 hectares de terres agricoles dans le schéma directeur de la Région Ile-de-France, la Région s'inscrivant ainsi en opposition au projet de l'Etat.

Depuis ce succès, les anciennes associations environnementales locales sont plus ou moins entrées en sommeil, alors que le rôle des acteurs de l'AMAP comme défenseurs des terres agricoles a été localement reconnu par l'obtention de financements auprès des collectivités locales (communes, conseil général). Ils ont notamment été soutenus pour la mise en place d'une ferme urbaine sur des terres agricoles non occupées et pour la réalisation d'un diagnostic sur la possibilité de mettre en place des circuits courts pour approvisionner la restauration collective locale. Pour ce dernier projet, l'association a embauché un consultant, choix qui témoigne d'un début de professionnalisation de cette organisation.

Sur ce territoire, ce sont donc les résidents qui se sont mobilisés en faveur de changements de pratiques, de modes de commercialisation et même de système agricoles - le développement des circuits courts supposerait en effet que les

\footnotetext{
6 Agriculteur et écrivain, Pierre Rabhi est un pionnier de l'agriculture biologique. Il est aujourd'hui reconnu expert international pour la sécurité alimentaire et a participé à l'élaboration de la Convention des Nations Unies pour la lutte contre la désertification. Il est l'initiateur du Mouvement pour la Terre et l'Humanisme.

7 Par la suite, l'agriculteur partenaire de l'AMAP a converti une cinquantaine d'hectares à l'agriculture biologique pour cultiver du blé. Une partie de ces terres accueillent également un volailler pratiquant l'agriculture biologique.
} 
agriculteurs, essentiellement des céréaliers, modifient profondément leur façon de travailler. Nous analyserons les stratégies d'intéressement construites par cette association et la façon dont elle s'appuie sur les enjeux liés à l'urbanisation du territoire et à la préservation des ressources naturelles.

\subsection{Une association d'usagers de l'eau mobilisée sur la réduction de l'utilisation des intrants}

Dans notre deuxième territoire, le bassin versant de l'Ancœur en Seine-et-Marne, nous avons étudié une animation, prise en charge par une association d'usagers de l'eau. Ce cas a été analysé à partir d'entretiens réalisés auprès d'agriculteurs (10), auprès de l'association d'usagers de l'eau (3) et à partir d'une observation de leurs interactions sur plusieurs mois. Suite à d'importants problèmes de diminution de la quantité d'eau disponible, en 1994, un comité de concertation entre les usagers de la nappe de Champigny, comprenant des industriels et des professionnels de l'eau, a été créé dans le but d'améliorer la connaissance et le suivi de la nappe. Les problèmes de quantité et de qualité de l'eau ayant été confirmés, une association est créée en 2001 avec le soutien financier du conseil général, du conseil régional et de l'Agence de l'eau. Aujourd'hui, l'association, employant 10 salariés et composée d'une quarantaine de membres ${ }^{8}$, a plusieurs missions sur son territoire de compétence comprenant 223 communes. Elle doit réaliser des diagnostics des pratiques phytosanitaires, identifier les zones vulnérables, élaborer un programme d'action visant le « 0 phyto » pour la direction départementale de l'équipement et de l'agriculture, protéger un site pilote d'engouffrement des eaux superficielles vers la nappe et le site d'extraction des calcaires de Champigny, et enfin informer et sensibiliser.

Un animateur agricole, ayant une formation en agronomie, travaille plus particulièrement sur le bassin versant de l'Ancœur qui comprend 12 communes. Depuis 2007, il a en charge l'accompagnement des agriculteurs contractualisant une mesure agri-environnementale territorialisée (MAET) à enjeu Eau - dans laquelle se sont engagés en 2008 dix agriculteurs sur un total de 70 sur le territoire. Cette MAET vise à accompagner, sur cinq ans, une réduction de la quantité de produits phytosanitaires utilisée par rapport à une référence de territoire en échange d'une rémunération ${ }^{9}$.

Le rôle de cette association est donc d'accompagner les agriculteurs vers la réduction d'intrants en organisant des réunions de suivi de la mise en place des MAET, réunions auxquelles sont conviés tous les acteurs de la filière agricole locale (chambre d'agriculture, coopératives,...). Elle a également une mission de sensibilisation technique effectuée en partenariat avec le conseiller "production intégrée" de la chambre d'agriculture de Seine-et-Marne, qui réalise des tours de plaine et des visites d'essais. Ces animations, sortes d'hybride entre des formes d'animation de type

8 Les services de l'Etat, les collectivités territoriales et intercommunalités, l'Union des maires du département, les gestionnaires de services d'eau, la profession agricole, le monde industriel, le monde associatif et des experts.

9 La rémunération est de $149 €$ par hectare engagée par an. 
associatif et des formes de conseil plus ancrées dans le monde agricole institutionnel, sont ouvertes aux agriculteurs du secteur n'ayant pas contractualisé la MAET Eau. Environ 25 agriculteurs au total suivent - à divers degrés - les réunions, et l'association continue à démarcher des agriculteurs.

\subsection{Une animation impulsée par un organisme agricole institutionnel}

Notre dernier terrain, l'Yerres, a été analysé à partir d'entretiens auprès d'agents de la chambre d'agriculture de Seine-et-Marne (4), d'agriculteurs (3) et de la participation à des réunions d'animation. Ce territoire, comprenant 21 communes, se situe également en Seine-et-Marne et les agriculteurs peuvent aussi y contractualiser la MAET Eau. L'animation du dispositif est assurée par la chambre d'agriculture, représentant par excellence du monde agricole institutionnel. Jusqu'à la fin des années 1990, le conseil de la chambre d'agriculture est diffusé dans le département à travers les groupes de développement agricoles (GDA), animés par des conseillers issus de la chambre d'agriculture et encadrés par la fédération départementale des syndicats d'exploitants agricoles (FDSEA). Suite à une réorganisation de son mode de fonctionnement, le syndicat a voulu imposer l'adhésion préalable des agriculteurs impliqués dans ces groupes. De nombreux agriculteurs ont alors quitté ces groupes, qui se sont peu à peu délités. De nouveaux groupes d'agriculteurs indépendants se sont formés, parfois animés par un conseiller privé (généralement d'anciens techniciens de chambre d'agriculture s'étant installés à leur propre compte). Cette reconfiguration a eu pour conséquence la perte d'ancrage territorial du conseil de la chambre d'agriculture et une perte de légitimité de ses conseillers auprès des agriculteurs du fait d'un manque de proximité. Dans ce contexte, il devient difficile aux conseillers de la chambre d'agriculture de diffuser de nouvelles pratiques auprès des agriculteurs:

"En terme de lien réel avec le terrain aujourd'bui la chambre n'a pas de réseau de connaissance et de lien fort avec les agriculteurs sur le terrain, il n'y en a plus. C'est la FDSEA qui faisait ce lien-là. » (animateur de l'Yerres)

Devant le peu de MAET contractualisées sur ce territoire (seulement trois agriculteurs en 2008 sur 148), l'animateur a fait le choix, comme nous le verrons, de s'appuyer sur les coopératives, plus en contact avec les agriculteurs que lui. En effet, celles-ci ont des essais implantés chez des agriculteurs et leur proposent certaines solutions techniques qui vont dans le sens de la réduction des intrants, comme par exemple l'invitation à remplacer certains insecticides chimiques par le lâché de prédateurs d'insectes nuisibles aux cultures.

On voit bien que sur chacun de ces trois territoires, les animateurs de changement agissent dans un contexte bien particulier qu'il faut prendre en compte pour comprendre les stratégies mises en œuvre pour mobiliser les agriculteurs sur la réduction des intrants. Nous allons maintenant analyser quels sont les leviers de conviction et d'action de ces animateurs de territoire et la manière dont ils construisent leurs stratégies d'intéressement. 


\section{Des activités d'animations diversifiées}

\subsection{Elaborer des stratégies d'intéressement}

Notre analyse nous permet de distinguer différentes stratégies d'intéressement développées par les animateurs qui reposent, en premier lieu, sur le recours à certains registres d'argumentation et, en deuxième lieu, sur l'emploi de dispositifs particuliers capables d'intéresser les agriculteurs à la réduction de l'usage des intrants de synthèse. Dans chacun des trois terrains, les animateurs développent des arguments divers, chacun ayant sa manière de "traduire " (Callon, 1986 ; Brives, 2006) le problème environnemental auprès des agriculteurs.

Les animateurs peuvent ainsi jouer les «prophètes de malheur » (Chateauraynaud et Torny, 1999) pour convaincre les agriculteurs du bien fondé d'un changement de pratiques en utilisant le registre de la peur et de la menace. Sur le plateau de Saclay, les acteurs justifient leurs injonctions par un argument écologique global - l'épuisement des ressources (terres agricoles, pétrole...) et la dégradation de la planète (réchauffement climatique, pollutions des sols et de l'eau...) - ce qui permet de relier l'injonction locale à l'avenir de la planète toute entière, dans un processus de montée en généralité, usuel dans les débats publics (Boltanski et Thévenot, 1991 ; Lafaye et Thevenot, 1993). C'est ce que nous révèle cet échange entre un agriculteur et un membre de l'association:

L'associatif: "L'intérêt des circuits courts (...) c'est de se dire, dans 15 ans, si on a des gros problèmes climatiques... on va les manger d'où, nos salades, si on ne les fait pas venir à des kilomètres autour ?! (...) Moi c'est mon métier de travailler là-dessus, et par exemple sur le prix du baril, moi je peux vous dire parce que je fais plein de conférences devant même des chefs d'entreprises. On leur parle d'un prix du baril à $300 \$$, il y a dix ans ça faisait rigoler tout le monde, maintenant plus personne ne rigole! (...). Il $y$ a des fermes qui explosent économiquement parce que l'énergie coûte trop cher (...).

L'agriculteur : On n'en sait rien, ça bouge tellement vite!»

La réaction de l'agriculteur aux propos de l'acteur associatif dans cette interaction nous montre que les deux types d'acteurs ne se situent pas dans un même univers cognitif. L'animateur associatif voit l'épuisement des ressources et leur renchérissement comme un phénomène linéaire inéluctable, alors que cet agriculteur - comme les autres rencontrés considère les prix des ressources comme sans cesse fluctuants, donc pouvant également baisser, ce qui ne l'incite pas à considérer un changement de pratiques. Il va sans dire que ce décalage ne facilite pas le développement commun d'un projet territorial.

En Seine-et-Marne, ce type d'argumentation reposant sur une menace d'ordre écologique, porté essentiellement par l'association d'usagers de l'eau, pourrait être plus efficace, étant donnés les importants problèmes liés à la qualité de l'eau et leur tangibilité (études et mesures locales sont là pour les étayer). Plusieurs auteurs ont pointé, sur les questions de risques sanitaires et environnementaux (Chateauraynaud, 2004), l'importance de cette notion de tangibilité : ainsi, le manque d'actualisation sous forme de dangers tangibles d'un risque considéré peut conduire à des postures attentistes, voire de négation - cas du réchauffement climatique, ou ci-dessus, de 
l'épuisement des ressources - sauf si sa propre intégrité corporelle est en jeu - cas, par exemple, des OGM pour les consommateurs (Lamine, 2003). Pourtant, même si la dégradation de la qualité de l'eau présente une certaine tangibilité, cet argument -au même titre que l'épuisement des ressources plus haut - ne semble pas directement influencer les pratiques agricoles à l'œuvre.

Dans ces conditions, les animateurs seine-et-marnais (issus de l'association et de la chambre d'agriculture) optent pour une argumentation davantage centrée sur une menace d'ordre réglementaire. Dans un département où divers dispositifs publics sont mis en œuvre pour l'amélioration de la qualité de l'eau et où le préfet se montre particulièrement actif dans cette perspective, cette argumentation peut en effet avoir un certain poids. Si les agriculteurs ne s'engagent pas dans la voie de la réduction de l'usage des intrants de synthèse tant que les dispositifs sont incitatifs (et non coercitifs), ils risquent d'être sanctionnés ou de voir le système agricole local s'effondrer si la réduction d'intrants devient une mesure contraignante.

"Il faut que l'ensemble des agriculteurs puisse avoir des moyens de répondre à ces enjeux. L'Etat pourrait aller très vite, il pourrait dire - et il le fait partiellement - il pourrait dire "je supprime telle molécule sur tel bassin, j'ai un problème de glyphosate, je l'interdis sur tel bassin, c'est terminé. Merci et au revoir" C'est ce qui a été fait en Bretagne, d'ailleurs, où ils limitent les doses d'azote à 160 unités. Et là (en Seine-et-Marne) on se retrouve avec des agriculteurs qui sont en production intensive en céréales, et qui sont en marché avec des clients des coopératives... Ça mettrait complètement par terre le système très rapidement. » (animateur de l'Yerres)

On voit, par ailleurs, se développer un autre registre argumentatif qui prend également des formes différentes selon les territoires: l'argument économique. En Seine-et-Marne, les animateurs évoquent souvent auprès des agriculteurs le fait que la réduction d'intrants peut être un moyen de réduire les charges financières de l'exploitation. Dans un contexte où les prix des céréales et des intrants sont très fluctuants, réduire sa consommation d'intrants peut être un moyen d'atteindre une certaine stabilité économique. La chambre d'agriculture s'est plus particulièrement positionnée sur ce créneau, comme en atteste le développement de références pour les systèmes «bas intrants » à partir de 2003 par un des conseillers techniques de l'organisme. Ces références ont par la suite été valorisées en lien avec l'exigence départementale d'amélioration de la qualité de l'eau. Sur le plateau de Saclay, les animateurs mettent également en avant la rentabilité économique des productions peu consommatrices en intrants de synthèse pour convaincre les agriculteurs de changer leurs pratiques agricoles, en invoquant, en outre, l'horizon de l'introduction croissante de l'alimentation d'origine biologique ${ }^{10}$ dans la restauration collective. Pour renforcer l'argument économique, les animateurs ont également recours à des dispositifs particuliers. En Seine-et-Marne, il s'agit de la MAET qui rémunère les changements de

10 Dans le projet de loi relatif à la mise en œuvre du Grenelle de l'environnement, «l'Etat se donne pour objectifs de recourir, pour l'approvisionnement de ses services de restauration collective, à des produits biologiques pour une part représentant 15\% des commandes en 2010 et 20\% en 2012 ». 
pratiques. Sur le plateau de Saclay, même si les acteurs associatifs ne disposent pas comme en Seine-et-Marne de mesures incitatives concernant directement la réduction des intrants, leur projet de mise en place de circuits courts fournissant des produits locaux et/ou issus de l'agriculture biologique à la restauration collective locale peut être vu comme un instrument incitatif économique indirect pour les changements de pratiques agricoles.

Enfin, en dernier lieu, se développent des dispositifs basés sur l'« exemplarité », qui consistent à donner accès aux agriculteurs à des exemples concrets des pratiques à mettre en œuvre au travers de "dispositifs de preuve" qui mettent en jeu "l'expérience sensible» (Bessy et Chateauraynaud, 1995). On les retrouve sur le plateau de Saclay et dans le bassin de l'Ancœur, à travers l'organisation de tours de plaine, de visites d'essais et la mise en relation des agriculteurs à intéresser avec d'autres agriculteurs ayant déjà changé leurs pratiques, tels que les agriculteurs biologiques. Cette stratégie s'appuie sur le fait que, pour un certain nombre d'agriculteurs, l'accès à un exemple sensible constitue l'une des meilleures façons de se renseigner sur une technique en particulier avant d'envisager de l'adopter, mais aussi sur le fait que l'adaptation locale de tel ou tel principe général constitue une des meilleures manières de juger de son intérêt (Henke, 2000).

Les animateurs ont donc recours à diverses stratégies d'intéressement reposant à la fois sur des arguments et des dispositifs soutenant concrètement leur action - diversité favorisée par la variété des acteurs impliqués dans l'animation. En effet, on note que les animateurs les plus distants du monde agricole sont susceptibles de développer des stratégies d'intéressement et des dispositifs originaux (menace de l'ordre écologique, instrument incitatif économique indirect) par rapport à des types d'incitations plus classiques (menaces d'ordre réglementaire, incitations économiques directes et exemplarité). Cependant, la diversité de l'animation ne se réduit pas à la capacité d'acteurs divers à développer des stratégies d'intéressement variées. La perspective comparative de notre travail nous permet également de mettre en avant l'importance de l'ancrage des animateurs pour la diffusion des pratiques agricoles peu consommatrices en intrants.

\subsection{Diffuser des pratiques innovantes et diversifiées : entre distance au monde agricole institutionnel et ancrage dans le monde agricole local}

La relation établie entre animateurs et agriculteurs participe des conditions de mise en œuvre de telle ou telle pratique ou de tel ou tel projet territorial. Cette relation et sa justesse reposent sur la capacité de l'animateur ou du conseiller à trouver la bonne distance à tenir avec l'agriculteur (Compagnone, 2006) et ainsi à gérer la tension entre la dimension sociale et la dimension technique de son activité (Lemery, 1994). Notre analyse, comparant différents types d'animateurs issus de structures plus ou moins à distance du monde agricole, nous porte à penser que l'établissement de cette juste relation ne repose pas seulement sur les compétences techniques mais également sur la construction d'un positionnement spécifique entre les exigences du développement agricole et les situations de terrain. Elle repose également sur le degré de distance de l'animateur au monde agricole institutionnel, qui influe sur sa capacité à proposer des 
pratiques innovantes par rapport aux techniques en place, et sur son ancrage dans le milieu agricole local qui se répercute sur son aptitude à diffuser ces pratiques innovantes. On constate en effet que la distance au monde agricole institutionnel permet aux animateurs de proposer des techniques très différentes de celles mises en pratique par les agriculteurs. En revanche, faire adopter ces techniques aux agriculteurs nécessite d'acquérir une certaine légitimité qui semble se fonder sur la capacité des animateurs à s'ancrer à la fois dans le monde agricole local et sur « le terrain » (Selznick, 1949).

L'association du plateau de Saclay, étrangère au monde agricole et qui justifie son intervention par un argumentaire souvent éloigné des préoccupations immédiates des agriculteurs, semble proposer des techniques très innovantes: comptage des vers de terre pour évaluer la qualité du sol, mise en culture de mélanges protéagineux-céréales, recours au «non-labour» dans un système relevant de l'agriculture biologique, etc., sans que cela ne corresponde nécessairement aux attentes ni aux réalités de l'agriculteur avec lequel elle coopère prioritairement via l'AMAP. Les animateurs de cette association, qui sont les consommateurs de l'AMAP, n'ayant pas de compétences techniques, font appel à des ressources extérieures et personnelles (contacts avec des chercheurs en agriculture, lectures personnelles) et se montrent très actifs et présents auprès de l'agriculteur. Cependant ils demeurent des acteurs non agricoles, qui seront peu impactés en cas d'échec des pratiques proposées. L'agriculteur en AMAP a pu ressentir le «conseil» de ces acteurs associatifs comme assez intrusif et loin d'une conception du conseil qui doit permettre aux agriculteurs de «porter leur choix sans pour autant prendre les décisions à leur place »(Compagnone, 2006). L'agriculteur a même limité les échanges sur les pratiques avec ses consommateurs-adhérents et, au moment de notre enquête, il ne tenait pas à s'engager catégoriquement et publiquement sur une transition vers l'agriculture biologique certifiée de la totalité de sa ferme. En partie pour des raisons économiques et d'organisation du travail mais aussi par crainte de leurs critiques s'il échoue ou abandonne - et ce alors même que ses productions de blé, lentilles et pommes de terres destinées à l'AMAP sont déjà conduites selon les principes de l'agriculture biologique et qu'il augmente régulièrement la surface de son exploitation cultivée selon ce mode de production.

En Seine-et-Marne, l'animateur de l'association des usagers de l'eau en Ancœur peut également proposer des techniques innovantes de façon plus libre que s'il travaillait pour un organisme institutionnel tel que la chambre d'agriculture, et donc élargir les choix possibles en termes de pratiques agricoles - même s'il tente de concilier au mieux objectifs de protection de l'eau et objectifs de production agricole. L'association invite les agriculteurs à participer aux formations à la production intégrée ${ }^{11}$ organisées par la

11 Les principes de la protection intégrée ont été développés à partir des années 1950 pour la production de fruits et de légumes par des chercheurs de l'Organisation internationale de lutte biologique contre les plantes et les animaux nuisibles (OILB), en lien avec des producteurs et conseillers. La production intégrée a été définie par l'OILB en 1993 comme "un système agricole de production (d'aliments etlou d'autres produits) utilisant les ressources et les mécanismes de régulation naturelle pour remplacer des apports dommageables à l'environnement tout en assurant une agriculture viable à long terme. " Dans le cas des grandes cultures, la protection intégrée correspond à la combinaison de réductions des intrants (pesticides et engrais) et de changements coordonnés de certaines pratiques censées favoriser les équilibres écologiques. 
chambre d'agriculture et qui sont d'ailleurs obligatoires en cas de souscription d'une MAET Eau. Mais elle propose également des visites chez des agriculteurs biologiques, des formations à des techniques considérées comme plus écologiques que les pratiques conventionnelles (semis sous couvert et semis direct), ainsi que la réalisation d'essais de désherbage mécanique et de réduction d'intrants chez les agriculteurs du bassin versant. Ici, par contraste avec le cas précédent, l'animateur bénéficie d'une certaine légitimité auprès des agriculteurs en raison de son appartenance au monde agricole -c'est un ancien conseiller technique d'une chambre d'agriculture bretonne. Il n'est donc pas «étranger » au monde agricole, il connaît bien son fonctionnement et la juste relation (Compagnone, 2006) qu'il convient de construire pour conseiller, ce qui, selon les agriculteurs, n'est pas toujours le cas de la part des « écolos » - terme souvent utilisé pour désigner ici les autres salariés de l'association d'usagers de l'eau. Pour accompagner les agriculteurs dans une démarche de changements de pratiques, il se base sur l'établissement d'une certaine proximité relationnelle (Hochereau, 2007) et donc sur un ancrage de terrain. Il passe en effet beaucoup de temps auprès des agriculteurs, leur téléphone fréquemment. Il connaît bien les caractéristiques sociales, professionnelles et familiales des agriculteurs et adapte son conseil en fonction de ses interlocuteurs. Il s'appuie également sur sa connaissance des réseaux informels et d'interconnaissance entre les agriculteurs, pour que les « leaders » du territoire relaient son action.

Sur le territoire de l'Yerres enfin, les relations entre la chambre d'agriculture et les agriculteurs se sont distendues suite à la disparition des groupes locaux de développement agricole. L'animateur de la chambre peut néanmoins s'appuyer sur les formations à la production intégrée qu'il organise pour rétablir une relation de conseil de proximité avec les agriculteurs. Si d'autres animations (formations à l'agriculture biologique, MAET Eau, ...) sont organisées depuis 2008 , le panel des interventions auprès des agriculteurs reste limité. En outre, l'animateur en charge du territoire reconnaît ne travailler qu'avec les agriculteurs déjà motivés. A une question concernant les moyens d'augmenter le nombre d'agriculteurs en MAET, il a ainsi répondu que les autres agriculteurs restent inaccessibles et qu'il ne les connaît pas vraiment. De fait, l'ancrage dans le monde agricole dont bénéficie la chambre d'agriculture ne lui suffit pas pour légitimer l'injonction d'un changement de pratiques, étant donné son absence d'ancrage sur le terrain. Face à cette difficulté, l'animateur a choisi de passer par l'intermédiaire des coopératives, en leur déléguant partiellement et de manière officieuse le pilotage de l'accompagnement au changement afin d'avoir un meilleur accès au terrain :

"On sait très bien que la chambre n'a pas les moyens de développer ça au niveau local. On sait qu'on n'a pas l'écoute au niveau des agriculteurs et que le seul moyen, c'est de passer par les coops. Enfin moi j’en suis persuadé. » (animateur de l'Yerres)

Mais les coopératives présentes sur ce territoire, bien qu'entretenant des relations de proximité avec les agriculteurs, ne valorisent pas la réduction de l'usage des intrants de synthèse et ne cherchent pas à proposer de nouveaux référentiels de conduites allant dans ce sens. Le changement de pratiques risque donc d'être limité à quelques agriculteurs pionniers. 
Cette description nous permet de relier le type de pratiques proposées par les animateurs à leur degré de distanciation par rapport au monde agricole institutionnel, ainsi que leur capacité à diffuser des pratiques peu consommatrices en intrants en fonction de leur ancrage dans le monde agricole et sur le «terrain». Nous avons synthétisé les différentes configurations de nos trois terrains dans la figure suivante:

Figure 1. Capacité à diffuser des pratiques peu consommatrices en intrants en fonction de l'ancrage et liberté de proposer des pratiques innovantes en termes de réduction d'intrants

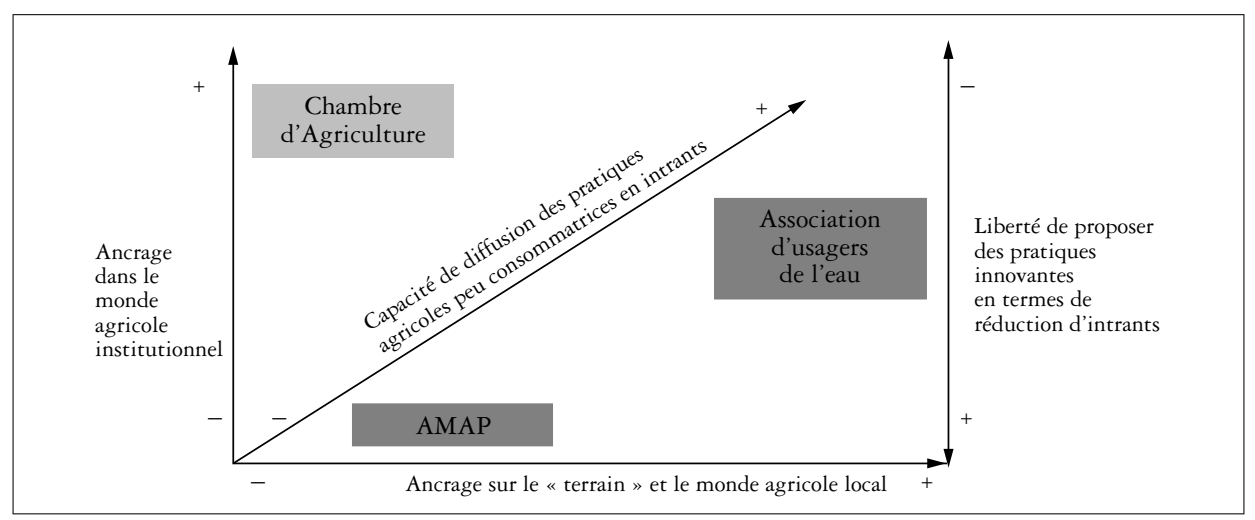

A travers cette figure, on peut voir comment, d'un côté, la chambre d'agriculture, organisme institutionnel agricole par excellence, voit sa liberté de proposition assez limitée et sa capacité de diffusion des pratiques affaiblie par son faible ancrage sur le terrain et le monde agricole local. A l'inverse, l'AMAP, très peu ancrée dans le monde agricole institutionnel possède une grande liberté de proposer des techniques innovantes en termes de réduction d'intrants et dispose d'une faible capacité à diffuser ces pratiques étant donné son faible ancrage dans le monde agricole local. L'association d'usagers de l'eau occupe, elle, une place intermédiaire dans la mesure où bien que relativement bien implantée dans le monde agricole, elle conserve une certaine liberté de proposition.

Ce schéma n'a pas pour vocation de hiérarchiser les différents organismes, mais plutôt de représenter leur marge de manœuvre quant à l'écart entre les techniques innovantes qu'ils proposent et les pratiques actuelles des agriculteurs de ces territoires et leur capacité à les diffuser.

\section{Quels effets sur les pratiques des agriculteurs?}

De quelles façons ces agriculteurs perçoivent-ils ces différentes stratégies d'intéressement, leurs pratiques évoluent-elles, et si oui, peut-on établir un lien avec ces stratégies ou ces évolutions semblent-elles indépendantes des actions menées? A partir de nos entretiens compréhensifs, nous avons reconstruit les trajectoires des agriculteurs en examinant non seulement les changements de pratiques et de conceptions au fil du temps, mais aussi le rôle des réseaux auxquels appartiennent les agriculteurs, de l'animation territoriale et du contexte local. Si certains agronomes 
analysent aussi les trajectoires d'exploitations (Capillon, 1993; Madelrieux et al., 2002), l'approche sociologique vise ici à explorer spécifiquement les changements de conceptions en parallèle des changements de pratiques et le rôle de diverses interactions sociales dans ces changements. Tous les agriculteurs rencontrés ont été en contact avec les animations territoriales présentées plus haut, mais tous n'ont pas nécessairement mis en œuvre des changements. Sur le plateau de Saclay, étant donné la taille réduite du territoire et le faible nombre d'agriculteurs encore en activité(8), l'enquête a été exhaustive. En Seine-et-Marne, dans le premier temps de notre enquête, les animateurs de territoire nous ont donné des contacts d'agriculteurs à rencontrer : agriculteurs chez qui des changements sont effectivement à l'œuvre dans l'Yerres (3); agriculteurs considérés comme potentiellement réceptifs mais n'ayant pas nécessairement modifié leurs pratiques dans l'Ancœur (10).

\subsection{Le rôle de la bio : un aiguillon en terme de changements des conceptions}

Lors des entretiens, nous n'avons volontairement pas cherché à orienter la discussion vers les questions environnementales pour les laisser émerger de manière spontanée dans les discours recueillis. Cependant, l'analyse textuelle du corpus d'entretiens ${ }^{12}$ montre que dans les propos des agriculteurs, «l'agriculture biologique » $(A B)$ est le thème qui revient le plus souvent après celui du «blé » - principale culture et objet de nos questions sur les changements de pratiques. Cette constatation est d'autant plus intéressante qu'aucun des agriculteurs rencontrés ne conduit toute sa production selon le cahier des charges de l'AB, même si l'agriculteur en AMAP du plateau de Saclay conduit les cultures de l'AMAP selon les principes de l'AB sans être certifié et que d'autres utilisent certaines techniques inspirées de l'AB.

Ainsi, les animations territoriales mais aussi la prégnance croissante des débats et discussions sur l'AB dans notre société semblent avoir un certain impact sur les conceptions des agriculteurs. La plupart des agriculteurs parlent d'AB et admettent en avoir aujourd'hui une meilleure perception quauparavant. Toutefois, il est probable que leurs propos soient en partie orientés par la présence d'un enquêteur s'intéressant aux réductions d'intrants, et en approfondissant l'analyse, on trouve des avis divers sur la question. Ces agriculteurs qui disent avoir de l'AB une meilleure image gardent tous une attitude défensive face à l'engouement qu'elle incarne: soit en mettant en avant le risque sanitaire, par exemple, lié aux mycotoxines dans le cas du blé, soit en soulignant le faible rendement de ce système de production par rapport à des systèmes plus conventionnels et qui pourrait conduire à l'avenir à des problèmes de sous-production et de famines. On trouve donc du côté des agriculteurs, comme de celui des défenseurs des systèmes respectueux de l'environnement rencontrés plus haut, des arguments

12 L'analyse textuelle a été réalisée à l'aide du logiciel Prospéro (PROgramme de Sociologie Pragmatique, Expérimentale et Réflexive sur Ordinateur), élaboré au sein du Groupe de sociologie pragmatique et réflexive (GSPR) et de l'association Doxa. Prospéro facilite la classification et le suivi de corpus ou d'ensembles thématiques de textes historiques ou diachroniques en langage naturel. Ainsi, ce logiciel permet de représenter et de comparer, dans leurs structures et leurs évolutions, les multiples formes d'expression et d'argumentation utilisées par les acteurs étudiés. 
relevant de la «prophétie de malheur»: aux uns, l'épuisement des ressources naturelles, aux autres, les risques de famine. Enfin, certains mettent en avant la faible rentabilité économique de l'agriculture biologique:

"Au point de vue économique, c'est sûr que ça ne marche pas! Vous avez rencontré beaucoup des gens qui faisaient de la culture bio, qui gagnaient leur vie? » (agriculteur)

Certains des agriculteurs se disent prêts à réduire leur utilisation d'intrants, mais ne voient pas l'AB comme un système encore suffisamment performant en termes de rendement et donc de rentabilité, ce qu'ils relient au manque de connaissances techniques disponibles mais aussi à leur propre manque de compétences. Certains reconnaissent également que les verrous ne sont pas seulement techniques mais culturels ou «psychologiques » : changer de système c'est aussi changer sa conception de l'exercice du métier (Lamine, 2011).

"C'est une remise en question complète (...) c'est vraiment une technique complètement différente, (...) moi un céréalier $100 \%$ de l'ancienne génération, qui a toujours en l'babitude d'avoir des champs nickel propre et tout, il faut vraiment qu'il change d'idée, on ne joue plus du tout dans la même cour, c'est plus du tout la même chose. » (agriculteur)

L'enquête montre donc que si ces agriculteurs sont encore loin d'envisager une transition vers l'AB, le déploiement de cette thématique sur la scène publique conduit chacun à se positionner par rapport à elle. Cela entraîne une reconnaissance croissante de l'AB par les agriculteurs pratiquant d'autres modes de production -inséparable toutefois d'une mise à distance critique de ce mode de production. Cependant, étant donné que notre enquête prend place dans des territoires fortement sensibilisés aux thématiques environnementales, il faudrait vérifier si cette reconnaissance est également présente chez des agriculteurs moins touchés par ce type d'animations.

\subsection{Vers une meilleure intercompréhension entre milieux agricoles et non agricoles?}

Ces animations, portées par des acteurs non agricoles, participent par ailleurs à améliorer l'intercompréhension entre agriculteurs et acteurs non agricoles. Lorsque des acteurs issus de la société civile non agricole (associations environnementales, riverains...) prônent une agriculture plus durable, ils pensent à l'agriculture biologique en raison de leur mauvaise connaissance d'autres formes de changement de pratiques. Les agriculteurs évoquent souvent cette «simplification » qui laisse peu de place à des avancées vers la réduction d'intrants, moins visibles qu'une conversion en bonne et due forme.

"C'est soit on met des produits et on est tout mawvais, soit on est bio et on est tout bon. » (agriculteur)

Or, des pratiques intermédiaires - entre agriculture conventionnelle et agriculture biologique - comme la production intégrée ou diverses stratégies de réduction d'intrants existent, même si elles sont encore difficilement valorisées et pour la plupart peu considérées par les acteurs du monde non agricole. Par ailleurs, il existe parfois un 
malentendu en ce qui concerne la réduction des intrants : les agriculteurs en parlent en termes d'impact, tandis que généralement les acteurs du monde non agricole s'intéressent plus à la réduction de l'usage. Ce décalage est aussi à l'origine de l'un des arguments critiques récurrents des agriculteurs conventionnels envers l'AB: cette dernière nécessite plus de passage d'instruments mécaniques - en particulier pour le désherbage - et donc plus de fioul, ce qui, malgré la suppression des intrants chimiques, remettrait en question selon eux son bilan environnemental.

Ce décalage entre les visions de l'agriculture provient, selon les acteurs rencontrés, d'un manque d'échange entre le monde agricole et non agricole. Certains agriculteurs dénoncent le fait que les acteurs non agricoles ne cherchent pas à comprendre leurs représentations.

"On veut des gens responsables qui se mettent à la place, il faut que chacun se mette à la place, (...) on est prêt à travailler avec ces gens-là, qui connaissent nos problèmes, mais des gens qui vont venir d'un autre univers et qui vont dire il faut qu'on casse les paysans, qu'on interdise tout, ce n'est pas possible. » (agriculteur)

Cependant, pour la plupart des agriculteurs rencontrés, cette difficulté à partager et échanger les points de vue - au sens propre du terme - est également causée par le manque d'ouverture et de transparence du monde agricole et donc le fait d'une responsabilité partagée.

"Je pense que c'est plus un problème de communication (...) en agriculture, on n'a jamais su communiquer, ni la profession, ni les agriculteurs, n'a su communiquer (...). Je pense qu'il y a une méconnaissance de la réalité. » (agriculteur)

Nous sommes là en présence d'un manque de communication au sens de Georges H. Mead (1963): des deux côtés, c'est peut-être la tentative de saisir l'attitude ou l'expérience d'autrui qui fait défaut.

La participation à une animation territoriale initiée par une structure non agricole, comme c'est le cas sur le plateau de Saclay et en Ancœur, est justement vue par beaucoup d'agriculteurs comme un moyen de mieux communiquer. L'inscription dans de tels dispositifs favorise des discussions entre les agriculteurs, leurs voisins urbains ou périurbains et les collectivités, et favorise une ouverture concrète à travers l'organisation de visites de leurs fermes. Un des premiers effets de ces animations est donc de rendre tangible l'activité agricole pour des acteurs non agricoles, ce qui contribue à faciliter les échanges et l'intercompréhension entre acteurs du monde agricole et non agricole et créer ainsi un espace de sens commun. Les difficultés et réalités de l'activité agricole constituent finalement pour tous les acteurs des éléments plus tangibles que des menaces parfois perçues comme exagérées par certains (par exemple, l'épuisement des ressources ou la dégradation de la qualité de l'eau). Cette meilleure interconnaissance et ces échanges permettent d'envisager un espace commun d'action, qui peut donner lieu à la construction de projets collectifs tels qu'une AMAP ou un système d'approvisionnement de la restauration collective locale. 


\subsection{La mise en pratique : verdissement ou écologisation?}

Notre enquête visait également à comprendre si l'animation territoriale, et notamment celle dispensée par des acteurs issus de la société civile non agricole (AMAP, association d'usagers de l'eau), produisait de réelles évolutions des pratiques agricoles ou si celles-ci restaient proches du référentiel conventionnel. Dans l'analyse, nous avons donc considéré toute forme de changement de pratiques au fil du temps et pas seulement ceux relatifs à la réduction d'intrants, afin de saisir au mieux la trajectoire d'évolution des pratiques agricoles et l'influence des animations territoriales. Dans notre approche qualitative, nous avons recensé les pratiques des agriculteurs allant dans le sens d'une réduction d'intrants (choix de variétés résistantes, réduction de dose d'intrants, réintroduction du labour pour lutter contre les adventices,...), afin d'évaluer la présence d'une dynamique de changement.

De manière générale, pour limiter les coûts d'exploitation dans un contexte de baisse des prix du blé et d'augmentation des prix des intrants sur le long terme, les agriculteurs s'attachent à ne plus utiliser des pleines doses et à «raisonner » mieux l'usage des intrants. Ces changements de pratiques s'inscrivent -comme cela a été démontré dans d'autres travaux (Compagnone, 2009) - dans le cadre d'une prise de distance par rapport aux conseils techniques traditionnels diffusés par les coopératives ou les entreprises phytosanitaires et vont de pair avec la réalisation d'un minimum d'observations ou d'essais personnels. Parmi les agriculteurs rencontrés, tous bricolent des pratiques «intermédiaires " de réduction d'intrants qui, bien que très diverses, semblent relever de l'un ou plusieurs de ces trois référentiels - la modulation de dose, les techniques de culture simplifiée et la protection intégrée - qui sont ainsi mobilisés par les agriculteurs dans des formes d'"hybridation technique " (Ansaloni et Fouilleux, 2006). Les pratiques sont «intermédiaires » en ce qu'elles se situent entre l'agriculture conventionnelle et l'agriculture biologique, dont on a vu qu'elle était aussi un référentiel au moins rhétorique dans les positions exprimées par les agriculteurs. D'autre part, on note que les agriculteurs "parlent » des changements de façon plus systémique ${ }^{13}$ à partir du moment où ils bénéficient des animations - par comparaison avec des agriculteurs non concernés par ces animations - notamment en évoquant les notions de processus agronomique ou de système de culture. L'exploitation est ainsi davantage vue comme un système complexe, au sein duquel peuvent être considérés des enjeux divers, comme par exemple les problématiques environnementales et les enjeux propres à tel ou tel territoire. Cette transformation est particulièrement à l'œuvre lorsque les agriculteurs bénéficient d'une animation relativement éloignée des institutions agricoles et qui envisage davantage son activité au niveau territorial et pas seulement au niveau agricole, comme c'est le cas sur le plateau de Saclay et dans le bassin de l'Ancœur. Cependant, la traduction concrète de cette vision systémique dans les pratiques n'est pas encore vraiment à l'œuvre, malgré la mise en place des pratiques

13 L'emploi du mot «systémique » vise ici à souligner que le changement n'est plus seulement envisagé par rapport à une technique isolée (par exemple, une suppression ou réduction des doses d'intrants sur une année ou une parcelle), mais se raisonne en termes de combinaison de plusieurs techniques, sur un pas de temps plus long, à l'échelle de toute l'exploitation. 
intermédiaires que nous avons évoquées. Pour faire évoluer leur système plus fortement, la plupart des agriculteurs ont tendance à temporiser en préférant attendre que des mesures coercitives soient mises en place (Lamine, 2011).

Toutefois, les effets spécifiques des animations territoriales n'émanant pas d'organismes agricoles en termes de changements de pratiques restent encore difficiles à évaluer. Il est difficile de dire que les animations observées aujourd'hui, mises en œuvre depuis au maximum six ans, sont toujours directement la cause des changements de pratiques identifiés. En Seine-et-Marne, la plupart des agriculteurs rencontrés ayant fait évoluer leurs pratiques sont impliqués depuis les années 1980 dans des réseaux professionnels prônant la réduction d'intrants comme les CETA ${ }^{14}$. Les animations considérées ont donc plus vraisemblablement conforté certaines évolutions en cours pour d'autres raisons (évolution des prix sur le long terme, influence d'autres réseaux). Le fait que la MAET Eau soit le plus souvent proposée aux agriculteurs déjà avancés dans leur réduction d'intrants, dans l'Yerres comme dans l'Ancœur, confirme cette hypothèse.

"En fait je me dis, on travaille toujours avec le même pool d'agriculteurs qui sont des gens qui sont bistoriquement engagés dans une réflexion, dans une stratégie, dans une démarche de progrès. » (animateur de l'Yerres)

Si dans le bassin de l'Ancœur, l'animateur se montre davantage proactif que dans l'Yerres et a su convaincre un plus grand nombre d'agriculteurs à souscrire à la MAET, il admet ne la proposer qu'à des agriculteurs qui lui semblent aptes à respecter le contrat facilement. On peut ainsi se demander si l'instrument d'incitation au changement ne se transforme pas en instrument de validation et s'il n'y a pas un décalage entre l'objectif initial et la mise en œuvre (Lascoumes et Le Galès, 2004). Sur le plateau de Saclay, les effets de l'animation sont peut-être plus significatifs dans la mesure où l'agriculteur a effectivement cessé d'utiliser des intrants de synthèse sur les légumes à destination des consommateurs de l'AMAP. Néanmoins, il faut noter que son épouse souhaitait qu'il se convertisse à l'agriculture biologique depuis déjà plusieurs années. L'intervention des «Amapiens » semble avoir été le déclencheur des changements de pratiques que l'agriculteur refusait d'effectuer auparavant; et donc d'une certaine manière l'animation a peut-être également permis ici d'encourager des évolutions latentes.

Même s'il n'est pas forcément directement l'effet de ces animations, le changement est toutefois bien à l'œuvre chez ces agriculteurs en matière de réduction des intrants. $\mathrm{Vu}$ sous un angle critique, celui-ci pourrait être qualifié pour l'instant comme un «verdissement» des pratiques agricoles, dans le sens où les agriculteurs adoptent seulement certains principes économes en intrants. Cependant, les animations territoriales présentent les changements d'une manière plus globale, en les reliant à un

14 Les centres d'études techniques agricoles (CETA) sont des associations d'agriculteurs, mettant en commun leurs expériences et des moyens financiers, dans le but d'améliorer leurs exploitations aux plans techniques, économiques et sociaux, et ce, de façon indépendante vis-à-vis des autres acteurs du monde agricole. Dans cette perspective, des réunions entre agriculteurs sont organisées et des intervenants extérieurs et privés sont souvent conviés en fonction des problèmes rencontrés par le groupe. 
intérêt général d'ordre environnemental et en proposant des systèmes de production comme l'agriculture biologique ou la production intégrée plutôt que des principes isolés. Pourront-elles alors favoriser, à l'avenir, un processus de redéfinition des pratiques relevant davantage d'une véritable "écologisation» des pratiques et des conceptions, à travers un renouvellement des modes de productions (Deverre et de Sainte Marie, 2008 ; Lamine et al., 2009) ? C'est ce que le suivi de ces changements sur des durées assez longues, au travers de dispositifs d'enquête adaptés, permettrait d'explorer, dans la suite de notre travail.

\section{Conclusion}

L'étude de ces trois dynamiques territoriales nous a montré la diversité des stratégies d'intéressement qui peuvent être mises en œuvre et la façon dont les agriculteurs se positionnent par rapport à ces stratégies. Il apparaît clairement que le degré de distance aux institutions agricoles influence l'activité de l'animateur de changement. La distance au monde agricole institutionnel permet une plus grande liberté de proposition de pratiques innovantes d'autant plus qu'elle affaiblit la possibilité d'être tenu pour responsable d'échecs éventuels. En revanche, on remarque que l'ancrage sur le terrain (liens concrets aux agriculteurs et connaissance de leur réseau) et l'expérience de l'agriculture (passage d'un animateur par une chambre d'agriculture dans un emploi antérieur) favorisent la diffusion des pratiques en légitimant l'activité de l'animateur.

L'enjeu, pour les différents animateurs, est donc de trouver une juste position qui leur permette de se construire une légitimité composite, reposant sur un dosage entre ancrage sur le terrain et dans le monde agricole local, distance au monde agricole institutionnel, reconnaissance des pratiques actuelles des agriculteurs, en vue de faire adopter des pratiques plus respectueuses de l'environnement. Comme l'ont montré des travaux antérieurs sur des questions à l'interface entre agriculture et environnement, telles que le recyclage agricole des boues d'épuration urbaine (Borraz et d'Arcimoles, 2003), il s'agit de définir un espace de sens commun avec les agriculteurs, permettant de dessiner un espace commun d'action et de coordination. Pour le cas du plateau de Saclay, si cet espace de sens commun semble assez solidement constitué autour des questions de résistance à l'urbanisation - le développement d'une agriculture de proximité et "propre » justifiant la préservation des terres agricoles - il est pour l'heure beaucoup plus fragile concernant les questions d'approvisionnement en circuits courts, sur lesquelles se centre pourtant l'animation étudiée. En Seine-etMarne, alors que l'on pouvait penser que la gestion de l'eau aurait pu constituer un espace de sens commun, il semble que celui-ci s'établisse davantage autour de la viabilité économique des exploitations et du respect des réglementations - la réduction de l'usage des intrants permettant à la fois d'atteindre une meilleure stabilité financière pour les agriculteurs, de préserver la qualité de l'eau et de respecter les normes. Si les deux ensembles d'objectifs sont pleinement cohérents avec les attentes environnementales actuelles, le premier (circuits courts) met en jeu des acteurs éloignés des agriculteurs (restauration collective, consommateurs, etc.) tandis que le deuxième (qualité de l'eau, réglementation et économie) est bien plus relié à des dispositifs incitatifs et coercitifs dont les agriculteurs sont familiers; ce qui leur donne une 
légitimité tout à fait différente. Cependant, force est de constater que c'est bien le type d'animation, les configurations dans lesquelles se trouvent l'animateur et sa capacité à ancrer son activité qui semblent faire la différence. En Seine-et-Marne, alors que sur les deux terrains considérés, les mêmes instruments coercitifs et incitatifs sont à l'œuvre, c'est dans le bassin de l'Ancœur, où l'animateur bénéficie d'un meilleur ancrage de terrain et d'une plus grande légitimité professionnelle que les agriculteurs évoquent les changements de pratiques dans une perspective davantage systémique et que l'on compte le plus de souscriptions de MAET. Sur le plateau de Saclay, l'agriculteur a changé ses pratiques. Malgré ses réticences quant à la manière dont est réalisée l'animation, celle-ci porte ses fruits de par sa vivacité et ses propositions innovantes.

Avec cette étude, on voit de quelles manières les formes de conseil agricole peuvent être renouvelées et ainsi, peut-être, mieux intégrer les contraintes environnementales. D'une part, la mise en place de démarches davantage territoriales et collectives basées sur la définition d'un espace de sens commun semble permettre de mieux considérer les problèmes environnementaux locaux - cette constatation allant dans le sens de travaux récents sur l'évolution du conseil agricole (Guillot et al., 2010). D'autre part, l'émergence d'acteurs non agricoles dans l'accompagnement au changement a aussi son importance. Leur activité participe d'un élargissement des possibles en termes de pratiques agricoles. Ils constituent également de nouveaux acteurs capables de mobiliser et convaincre les agriculteurs, en dehors du conseiller technique de la chambre d'agriculture, auquel est traditionnellement dévolu cette mission (Brives, 2006). Enfin, en donnant du sens aux changements de pratiques agricoles par rapport à des causes plus globales de protection de l'environnement, ces acteurs pourront être un facteur d'évolution des conceptions des agriculteurs et de « reconceptualisation » (Hill et MacRae, 1996) des systèmes agricoles conduisant à leur écologisation.

A ce titre, notre travail est une contribution aux débats sur l'évolution du conseil agricole et semble confirmer que la prise en compte de l'environnement en agriculture passe peut-être par une évolution du métier de conseiller agricole vers celui d'animateur territorial, qui permettrait une prise en charge globale du changement (Compagnone et Lemery, 2009).

\section{Bibliographie}

Akrich M., Callon M. et Latour B. (1988) A quoi tient le succès des innovations? 1 : L'art de l'intéressement, Gérer et comprendre, Annales des Mines 11, 4-17.

Ansaloni M., Fouilleux E. (2006) Changements de pratiques agricoles: facteurs et modalités d'hybridation technique des exploitations laitières bretonnes, Economie Rurale 292 (2), 3-17.

Barthe L. (2009) Le développement territorial dans les espaces ruraux, nouveaux défis des politiques publiques, in: Conseil et développement en agriculture: quelles nouvelles pratiques?, Compagnone C., Auricoste C. et Lémery B. (éds), Versailles, Quae, $167-185$. 
Bessy C., Chateauraynaud F. (1995) Experts et faussaires. Pour une sociologie de la perception, Paris, Metailié, 360 p.

Boltanski L., Thévenot L. (1991) De la justification: les économies de la grandeur, Paris, Gallimard, 483 p.

Borraz O., d'Arcimoles M. (2003) Réguler ou qualifier? Le cas des boues d'épuration urbaines, Sociologie du travail 45 (1), 45-62.

Brives H. (2006) Les conseillers agricoles et l'environnement: quelles compétences ?, in: Conseiller en agriculture, Rémy J., Brives H. et Lémery B. (éds), Dijon, Educagri/ INRA, 169-182.

Callon M. (1986) Eléments pour une sociologie de la traduction. La domestication des coquilles Saint-Jacques et des marins-pêcheurs dans la baie de Saint-Brieuc, L'Année sociologique 36, 169-208.

Candau J., Ruault C. (2005) Evolution des modèles professionnels en agriculture : scènes de débat, questions d'écologie et catégories de connaissances, Cahiers d'économie et de sociologie rurales $75,51-74$.

Capillon A. (1993) Typologie des exploitations agricoles, contribution à l'étude régionale des problèmes techniques, Thèse d'agronomie, INA-PG, Paris Grignon.

Chateauraynaud F. (2004) L'épreuve du tangible. Expériences de l'enquête et surgissements de la preuve, Raisons pratiques 15, 167-194.

Chateauraynaud F., Torny D. (1999) Les sombres précurseurs. Une sociologie pragmatique de l'alerte et du risque, Paris, Editions de l'EHESS, 476 p.

Compagnone C. (2009) Influence des réseaux de dialogues professionnels sur les changements des pratiques des viticulteurs, Colloque Mondiaviti, Forum des idées, 103-110.

Compagnone C. (2006) Le juste dans la relation de conseil en agriculture, in: Conseiller en agriculture, Rémy J., Brives $H$. et Lémery B. (éds), Dijon, Educagri/INRA, 221-234.

Compagnone C., Lemery B. (2009) Les nouvelles pratiques de développement: une exploration à poursuivre, in: Conseil et développement en agriculture : quelles nouvelles pratiques?, Compagnone C., Auricoste C. et Lémery B. (éds), Versailles, Quae, 235-242.

Deverre C., de Sainte Marie C. (2008) L'écologisation de la politique agricole européenne. Verdissement ou refondation des systèmes agro-alimentaires ?, Revue d'Etudes en Agriculture et Environnement 89 (4), 83-104.

Guillot M.-N., Olry P. et Cerf M. (2010) L'activité de conseil en grandes cultures : d'une épreuve à une autre, Colloque SFER Conseil en agriculture: acteurs, marchés, mutations, 12.

Henke C. R. (2000) Making a place for science: The field trial, Social Studies of Science $30(4), 483-511$. 
Hill S. B., MacRae R. J. (1996) Conceptual framework for the transition from conventional to sustainable agriculture, Journal of Sustainable Agriculture 7 (1), 81-87.

Hochereau F. (2007) L'enjeu d'expertise des acteurs locaux du développement durable. Le cas d'animateurs agricoles gestionnaires de risques environnementaux sur des bassins versants, Colloque Instituer le développement durable.

Lafaye C., Thevenot L. (1993) Une justification écologique? Conflits dans l'aménagement de la nature, Revue Francaise de Sociologie 34 (4), 495-524.

Lamine C. (2011) Anticiper ou temporiser. Injonctions environnementales et recompositions des identités professionnelles en céréaliculture, Sociologie du travail $53(1), 75-92$.

Lamine C (2005) Settling shared uncertainties: Local partnerships between producers and consumers, Sociologia Ruralis 45 (4), 324-345.

Lamine C. (2003) La construction des pratiques alimentaires face à des incertitudes multiformes, entre délégation et modulation. Le cas des mangeurs bio intermittents, Thèse de sociologie, EHESS, Marseille, Reprise sous le titre Les intermittents du bio, Paris-Versailles, MSH-Quae, (2008), 341 p.

Lamine C., Meynard J., Perrot N. et Bellon S. (2009) Analyse des formes de transition vers des agricultures plus écologiques : les cas de l'agriculture biologique et de la protection intégrée, Innovations Agronomiques 4, 483-493.

Lascoumes P., Le Galès P. (2004) Gouverner par les instruments, Paris, Presses de SciencesPo, $369 \mathrm{p}$.

Lémery B. (2006) Nouvelle agriculture, nouvelles formes d'exercice et nouveaux enjeux du conseil aux agriculteurs, in: Conseiller en agriculture, Rémy J., Brives $\mathrm{H}$. et Lémery B. (éds), Dijon, Educagri/INRA, 238-252.

Lémery B. (2003) Les agriculteurs dans la fabrique d'une nouvelle agriculture, Sociologie du travail 45 (1), 9-25.

Lémery B. (1994) Une position d'expert incertaine: les conseillers techniques en agriculture, in: Pairs et experts dans l'agriculture. Dialogues et production de connaissance pour l'action, Darré J.-P. (éd.), Toulouse, Erès, 91-116.

Madelrieux S., Dedieu B. et Dobremez L. (2002) Modifications de l'utilisation du territoire lorsque des éleveurs cherchent à résoudre leurs problèmes de travail, Fourrages 172, 355-368.

Mead G. (1963) L'esprit, le soi et la société, Paris, PUF, 434 p.

Quermonne J. (1986) Les régimes politiques occidentaux, Paris, Seuil, 316 p.

Ruault C. (2006) Le conseil aux agriculteurs « bios » : un analyseur des interrogations et des évolutions du conseil en agriculture, in: Conseiller en agriculture, Rémy J., Brives H. et Lémery B. (éds), Dijon, Educagri/INRA, 184-204.

Selznick P. (1949) TVA and the Grass Roots, Berkley, University of California Press, $287 \mathrm{p}$. 